\title{
Conciliation de l'obligation de neutralité religieuse et du principe de non-discrimination en droit de la fonction publique
}

\section{Olivia Bui-Xuan}

\section{(2) OpenEdition}

\section{Journals}

Édition électronique

URL : https://journals.openedition.org/rdr/673

DOI : $10.4000 /$ rdr.673

ISSN : 2534-7462

Éditeur

Presses universitaires de Strasbourg

\section{Édition imprimée}

Date de publication : 31 octobre 2017

Pagination : 33-50

ISBN : 978-2-86820-974-0

ISSN : 2493-8637

\section{Référence électronique}

Olivia Bui-Xuan, «Conciliation de l'obligation de neutralité religieuse et du principe de non-

discrimination en droit de la fonction publique », Revue du droit des religions [En ligne], 4 | 2017, mis en ligne le 15 janvier 2020, consulté le 23 mai 2022. URL : http://journals.openedition.org/rdr/673 ; DOI https://doi.org/10.4000/rdr.673 


\section{CONCILIATION DE L'OBLIGATION DE NEUTRALITÉ RELIGIEUSE ET DU PRINCIPE DE NON-DISCRIMINATION EN DROIT DE LA FONCTION PUBLIOUE}

\section{Olivia BUI-XUAN}

Université d'Évry-Val-d'Essonne, Centre de recherche Léon Duguit (CRLD)

\section{RÉSUMÉ}

L'articulation de l'obligation de neutralité religieuse des agents publics et du principe de non-discrimination à raison de la religion apparaît de plus en plus délicate en droit de la fonction publique. L'interprétation extensive dont fait l'objet le devoir de neutralité religieuse paraît avoir pour conséquence de reléguer au second plan le principe de non-discrimination. Si la primauté de l'obligation de neutralité religieuse sur le principe de non-discrimination à raison de la religion peut se justifier face à des comportements intégristes, elle semble générer un désavantage pour les personnes de confession musulmane.

\section{ABSTRACT}

The articulation of the obligation of religious neutrality and the principle of non-discrimination is becoming more and more difficult in public service law. The extensive interpretation of the duty of religious neutrality appears to have the consequence of relegating the principle of non-discrimination to the background. While the primacy of the obligation of religious neutrality on the principle of non-discrimination can be justified in the face of fundamentalist behavior, it appears to generate a disadvantage for Muslims. 
u premier abord, l'obligation de neutralité religieuse et le principe de
non-discrimination semblent participer du même mouvement : la neutralité serait garante de la non-discrimination entre les religions. Il existerait même une imbrication des principes de neutralité et de non-discrimination, tout comme on constate un lien étroit entre principes de laïcité et d'égalité. L'article $1^{\text {er }}$ de la Constitution de 1958 ne dispose-t-il pas : "La France est une République indivisible, laïque, démocratique et sociale. Elle assure l'égalité devant la loi de tous les citoyens sans distinction d'origine, de race ou de religion. Elle respecte toutes les croyances »?

Certes, principes d'égalité et de non-discrimination ne se recouvrent pas totalement. Si la doctrine n'est pas unanime quant aux liens que ces principes entretiennent ${ }^{1}$, certains auteurs les estiment aujourd'hui «interchangeables ${ }^{2}$ " et nombreux sont ceux qui ont constaté un "passage de l'égalité à la non-discrimination ${ }^{3} »$. Nous considérons, pour notre part, que le principe de non-discrimination constitue une déclinaison du principe d'égalité, dont l'importance s'est accrue dans les années 2000, sous l'influence du droit communautaire, sensible à l'égalité réelle, comme l'attestent notamment les lois du 16 novembre $2001^{4}$ et du 27 mai $2008^{5}$. Actuellement, en droit de la fonction publique, le principe d'égalité est largement (mais pas exclusivement) appréhendé à travers le prisme du principe de non-discrimination ${ }^{6}$.

Les relations entre principe de laïcité et principe d'égalité (ou de nondiscrimination) sont par ailleurs régulièrement interrogées. Déjà, en 1999, Pierre-Henri Prélot constatait une « inégalité des religions devant la $\operatorname{loi}^{7}$ ». Marie-Joëlle Redor-Fichot soutenait pour sa part que « le principe de laïcité doit assurer l'égal respect de toutes les croyances et non-croyances; en ce sens il est une garantie de la non-discrimination entre les croyances. Mais

1. V. Edel F., « Le chaos des interprétations du principe d'égalité ou de non-discrimination », Droits, 2015/1, p. 117.

2. Ibid., p. 139 ; Woenrling J.-M., « Le droit français de la lutte contre les discriminations à la lumière du droit comparé ", Informations sociales, n 148, 2008, p. 58.

3. V. par ex. Pelloux R., "Les nouveaux discours sur l'inégalité et le droit public français », RDP 1982, p. 913.

4. Loi $\mathrm{n}^{\circ}$ 2001-1066 du 16 novembre 2001 relative à la lutte contre les discriminations.

5. Loi n ${ }^{\circ} 2008-496$ du 27 mai 2008 portant diverses dispositions d'adaptation au droit communautaire dans le domaine de la lutte contre les discriminations.

6. V. Toubon J., «Lutter contre les discriminations et promouvoir l'égalité dans l'emploi public : l'action du défenseur des droits », RFAP 2015, p. 183-194 ; L'HORTY Y., Les discriminations dans l'accès à l'emploi public, Rapport au Premier ministre, juin 2016.

7. Prélot P.-H., «Les religions et l'égalité en droit français », Les Cahiers de droit, $\mathrm{n}^{\circ} 404$, 1999, p. 853. 
cette neutralité nécessaire à l'égale liberté de conscience est remise en cause si une croyance est favorisée au détriment des autres, ou si une croyance est au contraire stigmatisée ${ }^{8} »$.

Depuis quelque temps, en droit de la fonction publique, la conciliation de l'obligation de neutralité religieuse - qui découle directement du principe de laïcité - et du principe de non-discrimination - en tant que déclinaison du principe d'égalité - apparaît de plus en plus délicate. Il est même permis de se demander si, dans certains cas, l'interprétation qui est faite de l'obligation de neutralité religieuse ne génère pas des discriminations, c'est-à-dire des différences de traitement prohibées, à raison de la religion. On observe en effet que les pouvoirs publics cherchent à consolider la neutralité religieuse des fonctionnaires. La portée juridique de cette obligation a notamment été renforcée par la loi du 20 avril $2016^{9}$ : alors que l'obligation de neutralité des agents publics était jusqu'à présent jurisprudentielle, elle trouve dans ce texte, pour la première fois, un fondement législatif.

Le nouvel article 25 de la loi du 13 juillet 1983 portant droits et obligations des fonctionnaires dispose désormais que «Dans l'exercice de ses fonctions, [le fonctionnaire] est tenu à l'obligation de neutralité » et qu'il « exerce ses fonctions dans le respect du principe de laïcité. À ce titre, il s'abstient notamment de manifester, dans l'exercice de ses fonctions, ses opinions religieuses ». Il y est par ailleurs précisé que « Le fonctionnaire traite de façon égale toutes les personnes et respecte leur liberté de conscience et leur dignité ${ }^{10} »$.

La loi du 20 avril 2016 intègre ainsi la notion de laïcité dans le statut général de la fonction publique. De façon classique, la laïcité à laquelle il est fait référence implique tant la neutralité religieuse que la liberté de conscience des fonctionnaires. Cette dernière rejoint la liberté d'opinion, garantie à l'article 6 de la loi du 13 juillet 1983. Depuis 2001, celui-ci précise par ailleurs qu'« Aucune distinction, directe ou indirecte, ne peut être faite entre les fonctionnaires en raison de leurs opinions politiques, syndicales, philosophiques ou religieuses $[\ldots] »$.

En droit de la fonction publique, la liberté d'opinion des agents publics est ainsi étroitement liée au principe de non-discrimination. Elle consiste

8. Redor-Fichot M.-J., « Laïcité et principe de non-discrimination », CRDF, n 4, 2005, p. 87.

9. Loi n ${ }^{\circ} 2016-483$ du 20 avril 2016 relative à la déontologie et aux droits et obligations des fonctionnaires.

10. Ibid. Cet article indique également qu'« Il appartient à tout chef de service de veiller au respect de ces principes dans les services placés sous son autorité ». 
essentiellement à leur offrir la garantie de n'être l'objet d'aucune discrimination en lien avec l'usage qui est fait de cette liberté ${ }^{11}$. L'articulation de l'obligation de neutralité religieuse et de l'interdiction de discrimination à raison de la religion peut toutefois s'avérer problématique, notamment dans un contexte où " les demandes communautaires se multiplient ${ }^{12}$ ", constat qui a motivé l'installation, en juin 2016, de la Commission laïcité et fonction publique, présidée par Émile Zuccarelli.

L'intégration du principe de laïcité dans le statut général de la fonction publique est loin d'être anodine. Dans un contexte de montée des communautarismes, elle vise principalement à contrer les pratiques des agents publics ou candidats à la fonction publique qui ne respecteraient pas, à différents degrés, le devoir de neutralité religieuse. Essentiellement quatre problèmes ont été observés par la commission Zuccarelli : "l'interaction femmes/hommes (qui peut se retrouver notamment dans la question de la salutation, ou dans le refus de respecter l'autorité hiérarchique lorsque le supérieur est de l'autre sexe), le port de vêtements ou de signes religieux, les demandes d'autorisation d'absence et, plus rarement, la pratique religieuse sur le lieu de travail ${ }^{13}$." Le rapport ne distingue toutefois pas les réponses à apporter à ces "situations problématiques ». S'il précise que leur nombre « reste faible dans la plupart des structures publiques ${ }^{14}$ ", il indique qu' « au moment du recrutement des agents publics (y compris des contractuels), il est nécessaire que l'exigence de neutralité qui incombe à tout agent public soit systématiquement rappelée au candidat ${ }^{15}$ » et préconise, pour tous les agents publics, une formation en matière de laïcité, laissant penser que la consolidation du devoir de neutralité constitue le remède aux difficultés constatées. La circulaire du 15 mars 2017 relative au respect du principe de laicité dans la fonction publique participe de la même logique ${ }^{16}$.

Le renforcement juridique de l'obligation de neutralité religieuse des agents publics s'inscrit dans le contexte post-attentats de janvier 2015 : parmi les 60 mesures proposées lors du Comité interministériel à l'égalité et à la

11. CE, sect, 28 févr. 2001, Casanovas, Rec., p. 107, RFAP 2001, p. 399, concl. Fombeur P.

12. Propos de la ministre de la Fonction publique, Annick Girardin, relatés à l'AJDA 2016, p. 1154.

13. Laïcité et fonction publique, rapport de la commission présidée par Émile Zuccarelli, déc. 2016, p. 14.

14. Ibid., p. 5.

15. Ibid., p. 24.

16. Circulaire de la ministre de la Fonction publique du 15 mars 2017 relative au respect du principe de laïcité dans la fonction publique (NOR : RDFF1708728C), p. 1. 
citoyenneté (CIEC) du 6 mars 2015, figure la « réaffirmation de la laïcité comme une valeur fondamentale de la fonction publique ». Le rapport du CIEC du 26 octobre 2015 se félicite, quant à lui, que « la mesure "consacrer le principe de laïcité dans le statut général comme valeur fondamentale de la fonction publique" [ait] été intégrée dans le projet de loi relatif à la déontologie et aux droits et obligations des fonctionnaires ${ }^{17}$ ».

Alors que certains médias se font l'écho de «comportements de transgression du principe de laïcité ${ }^{18} »$ dans la fonction publique, comme l'aménagement de salles de prières ou le refus de salutation des femmes, tout se passe alors comme si la laicité était pensée comme un instrument de prévention de la radicalisation ${ }^{19}$. Lutte contre la radicalisation et laïcité sont même amalgamées dans des revues destinées aux agents publics où des dossiers sur l'islamisme fleurissent ${ }^{20}$. Les rapports et autres plans ou chartes mêlant la laïcité à la prévention de la radicalisation se multiplient également dans différents domaines ${ }^{21}$, avec un risque de stigmatisation des musulmans.

On constate cependant que dès le début des années 2000 - donc indépendamment du développement de l'intégrisme et de la radicalisation islamistes ${ }^{22}$, mais au moment où le port du voile commence à être appréhendé comme

17. Rapport du CIEC La République en actes, 26 oct. 2015, p. 16.

18. V. par ex. «Paris : des cas de radicalisation à l'Hôtel de Ville », Le Parisien, 27 nov. 2015 : http://www.leparisien.fr/paris-75/paris-des-cas-de-radicalisation-a-l-hotel-deville-27-11-2015-5318989.php ou «Radicalisation : la police n'est pas épargnée », Le Parisien, 9 mars 2016 : http://www.leparisien.fr/faits-divers/radicalisation-la-police-n-estpas-epargnee-09-03-2016-5610617.php [consultés le 26 juin 2017].

19. Selon Farhad Khosrokhavar, «Par radicalisation, on désigne le processus par lequel un individu ou un groupe adopte une forme violente d'action, directement liée à une idéologie extrémiste à contenu politique, social ou religieux, qui conteste l'ordre établi sur le plan politique, social ou culturel », Khosrokhavar F., Radicalisation, Paris, Éd. MSH, 2015.

20. V. par ex. le dossier présenté sur le site de La Gazette des communes en décembre 2015 : « «Islamisme : les services des collectivités territoriales sont-ils touchés ? » : http://www. lagazettedescommunes.com/421507/islamisme-les-services-des-collectivites-territorialessont-ils-touches/ ou le $\mathrm{n}^{\circ} 558$ de Gestions hospitalières de septembre 2016 «Fonction publique et radicalisation. Un risque de moins en moins théorique $»:$ http://gestionshospitalieres.fr/fonction-publique-et-radicalisation/ [consultés le 26 juin 2017].

21. Thierry M., Valeurs républicaines, laïcité et prévention des dérives radicales dans le champ du travail social, Rapport à la ministre des Affaires sociales, de la Santé et du Droit des femmes, 2016 ; Conseil régional d'Île-de-France, délibération n ${ }^{\circ}$ CR 143-16 du 8 juillet 2016 portant Plan régional de lutte pour la défense de la laïcité, les valeurs de la République et l'engagement dans la prévention de la radicalisation; Charte régionale des valeurs de la République et de la laïcité adoptée en mars 2017 par le Conseil régional d'Île-de-France, délibération $n^{\circ}$ CR 2017-51.

22. Le Larousse définit l'intégrisme comme « l'attitude et la disposition d'esprit de certains croyants qui, au nom du respect intransigeant de la tradition, se refusent à toute évolution ». À la différence de la radicalisation, l'intégrisme n'est pas violent. 
un problème politique ${ }^{23}$-, le juge administratif a retenu une interprétation extensive de l'obligation de neutralité des agents publics. On peut à ce titre se demander si une telle interprétation ne crée pas une différence de traitement au détriment des fonctionnaires ou futurs fonctionnaires musulmans, dans la mesure où ce sont principalement les personnes de confession musulmane qui en pâtissent.

Face aux lectures intégristes de l'islam, on peut trouver acceptable de faire primer le devoir de neutralité religieuse des agents publics sur le principe de non-discrimination à raison de la religion (1). Pour autant, la relégation au second plan du principe de non-discrimination que l'on observe depuis une quinzaine d'années paraît avoir des répercussions négatives sur l'ensemble des musulmans, y compris les non-intégristes (2).

\section{UNE PRIMAUTÉ DE L'OBLIGATION DE NEUTRALITÉ RELIGIEUSE SUR LE PRINCIPE DE NON-DISCRIMINATION ACCEPTABLE FACE AUX PRATIQUES INTÉGRISTES}

Avant de s'interroger sur l'application qui est faite du principe de nondiscrimination à raison de la religion aux musulmans retenant une conception intégriste de l'islam (1.2), il convient de rappeler que le principe de nondiscrimination à raison de la religion reste essentiel en droit de la fonction publique (1.1).

\subsection{FONDEMENTS ET MISE EN OEUVRE DU PRINCIPE DE NON-DISCRIMINATION À RAISON DE LA RELIGION}

Le principe de non-discrimination à raison de la religion découle du principe d'égalité ${ }^{24}$ et, s'agissant des candidats à la fonction publique, du principe d'égale admissibilité aux emplois publics.

Si l'on s'en tient aux sources du droit interne, l'article $1^{\text {er }}$ de la Constitution de la $V^{e}$ République dispose que la France «assure l'égalité devant la loi de tous les citoyens sans distinction d'origine, de race ou de religion »,

23. Auquel est censée répondre la loi no 2004-228 du 15 mars 2004 encadrant, en application du principe de laïcité, le port de signes ou de tenues manifestant une appartenance religieuse dans les écoles, collèges et lycées publics.

24. Pour Alexis Zarca, « le principe d'égalité offre une protection contre certaines discriminations », ZARCA A., L'égalité dans la fonction publique, Bruxelles, Bruylant, 2014, p. 33. 
l'article 6 de la Déclaration des droits de l'homme et du citoyen affirme que « tous les citoyens [...] sont également admissibles à toutes dignités, places et emplois publics, selon leur capacité, et sans autre distinction que celle de leurs vertus et de leurs talents », et le préambule de la Constitution de 1946 indique, à son alinéa 5, que «nul ne peut être lésé, dans son travail ou son emploi, en raison de ses origines, de ses opinions ou de ses croyances». L'article 6 de la loi du 13 juillet 1983 précise, quant à lui, qu'« aucune distinction, directe ou indirecte, ne peut être faite entre les fonctionnaires en raison de leurs opinions $[\ldots]$ religieuses $[\ldots] »$.

Le Conseil d'État a consacré la source constitutionnelle du principe d'égale admissibilité aux emplois publics dans l'arrêt Bleton de $1988^{25}$. Celui-ci met l'accent sur une sélection des fonctionnaires sur le seul fondement de leurs mérites et de leurs compétences. Aussi l'intervention d'autres critères dans la sélection des fonctionnaires est-elle en principe interdite.

C'est ainsi que les fonctionnaires ne peuvent pas, à l'occasion de leur recrutement, faire l'objet d'une discrimination fondée sur leurs croyances religieuses. Dans l'arrêt Bouteyre de $1912^{26}$, le juge administratif a cependant rejeté le recours d'un ecclésiastique demandant l'annulation du refus du ministre de l'Instruction publique de l'admettre sur la liste des candidats à un concours d'agrégation de l'enseignement secondaire. Pour le commissaire du gouvernement Heilbronner, « l'autorité qui fait la nomination a [...] forcément, dans l'intérêt du service que la fonction a pour but d'assurer, à exercer un certain pouvoir d'appréciation sur les mérites des candidats ».

Dans l'arrêt Beis de 1939²7, le Conseil d'État a en revanche annulé la décision d'un inspecteur d'académie qui avait écarté une candidature à un emploi d'institutrice au motif que la candidate avait poursuivi ses études dans des établissements d'enseignement confessionnels et ne présentait dès lors pas les garanties nécessaires de laïcité pour exercer dans l'enseignement public. De même, dans l'arrêt El Haddioui de $2009^{28}$, il a estimé contraire au principe d'égal accès aux emplois publics le fait qu'un jury ait lourdement interrogé un candidat sur ses origines et ses pratiques confessionnelles ainsi que sur celles de son épouse.

25. CE, ass., 16 déc. 1988, n 77713, Bleton, Rec. CE, p. 451, AJDA 1989, p. 102, concl. Vigouroux Ch.

26. CE, 10 mai 1912, Abbé Bouteyre, Rec. CE, p. 561, concl. Heilbronner; GAJA, n 25.

27. CE, sect., 25 juill. 1939, Beis, Rec. CE, p. 524.

28. CE, 10 avr. 2009, n 311888, El Haddioui, Rec. CE, p. 158, AJDA 2009, p. 1386, note Calvès G. ; JCP A 2009, p. 2108, note Jean-Pierre D. 
Depuis 2001, sous l'influence du droit communautaire, le statut général de la fonction publique a en outre intégré l'interdiction de la discrimination indirecte $^{29}$, définie par la loi du 27 mai 2008 comme « une disposition, un critère ou une pratique neutre en apparence, mais susceptible d'entraîner, pour l'un des motifs mentionnés au premier alinéa ${ }^{30}$, un désavantage particulier pour des personnes par rapport à d'autres personnes, à moins que cette disposition, ce critère ou cette pratique ne soit objectivement justifié par un but légitime et que les moyens pour réaliser ce but ne soient nécessaires et appropriés ».

Par ailleurs, le principe de non-discrimination à raison de la religion ne s'applique pas uniquement au stade du recrutement; il vaut tout au long de la carrière des fonctionnaires. Cela engendre un certain nombre de conséquences sur le contenu de leur dossier : l'article 18 de la loi du 13 juillet 1983 indique ainsi qu'«il ne peut être fait état dans le dossier d'un fonctionnaire, de même que dans tout document administratif, des opinions ou des activités politiques, syndicales, religieuses ou philosophiques de l'intéressé ».

Le principe de non-discrimination à raison de la religion est donc régulièrement rappelée ${ }^{31}$. Il est permis de se demander comment il est appliqué aux agents publics ou candidats fonctionnaires retenant une conception intégriste de leur religion.

\subsection{COMMENT APPLIQUER LE PRINCIPE DE NON-DISCRIMINATION À RAISON DE LA RELIGION AUX AGENTS PUBLICS OU CANDIDATS FONCTIONNAIRES INTÉGRISTES ?}

L'exemple du jugement du tribunal administratif de Lyon en date du 17 juin $2015^{32}$ est, à cet égard, instructif. Le juge administratif a rejeté le recours d'un candidat à la fonction publique dont la candidature avait été écartée au motif qu'il avait l'intention de manifester ses croyances religieuses

29. Article 6 de la loi $n^{\circ} 83-634$ du 13 juillet 1983 portant droits et obligations des fonctionnaires.

30. Soit les critères prohibés par l'article 6 de la loi du 13 juillet 1983.

31. Le rapport annuel 2015-2016 de l'Observatoire de la laïcité indique qu'« est strictement interdite toute discrimination (lors du recrutement comme en cours de la carrière) en raison notamment de l'appartenance ou non-appartenance à une religion ou d'une pratique religieuse, à titre privé » : http://www.gouvernement.fr/rapport-annuel-de-l-observatoirede-la-laicite-2015-2016-5100 [consulté le 26 juin 2017].

32. TA Lyon, $8^{\mathrm{e}}$ ch., 17 juin $2015, \mathrm{n}^{\circ}$ 1204943, AJFP 2016, p. 28, concl. LAVAL J.-S. 
dans le cadre de son service. Plus précisément, durant l'épreuve orale, le candidat avait lui-même indiqué qu'en raison de sa religion, il refusait de serrer la main des femmes et souhaitait prier pendant ses pauses.

On doit ici rappeler que le principe de non-discrimination interdit au jury, lui-même soumis à un devoir de neutralité et d'impartialité vis-à-vis des candidats, d'orienter l'oral de recrutement dans le but de favoriser ou de défavoriser tel ou tel groupe confessionnel. On peut dès lors se demander si les membres du jury méconnaissent leurs obligations dans l'hypothèse où leur décision prend en compte la volonté du candidat de s'isoler et de ne pas respecter, en vertu de sa religion, certaines normes juridiques, mais également sociales.

$\mathrm{Si}$, en soi, le refus de serrer la main à des collègues ne semble pas pouvoir être retenu comme motif de rejet d'une candidature, le caractère systématique de ce mode de conduite à l'égard des femmes montre que le candidat n'a pas intégré le principe d'égalité entre les sexes et demandera peut-être des aménagements, s'il ne souhaite par exemple pas conduire un bus dont le volant a préalablement été touché par une femme. On peut dès lors comprendre que des considérations relatives au bon fonctionnement du service et même, dans certains cas, à l'ordre public soient susceptibles de venir limiter le principe de non-discrimination à raison de la religion. Il semble pourtant insatisfaisant d'écarter la candidature d'une personne compétente en se fondant sur son comportement hypothétique. Ce raisonnement suscite en effet plusieurs questions.

Si on décide de ne pas recruter de personnes retenant une lecture intégriste d'une religion, la première question qui se pose est celle de savoir quelle définition juridique on retient de l'intégrisme religieux. Autrement dit, sur quels critères se fondent les membres d'un jury et le juge administratif pour affirmer qu'une personne est intégriste? En l'état actuel du droit, si le législateur ${ }^{33}$ et le juge administratif ${ }^{34}$ sont régulièrement confrontés à la question de l'intégrisme religieux, il n'existe aucune définition juridique de ce fait social. Par suite, il est difficile de savoir quelles pratiques et comportements peuvent recevoir une telle qualification. Certes, la lutte

33. Loi $\mathrm{n}^{\circ}$ 2010-1192 du 11 octobre 2010 interdisant la dissimulation du visage dans l'espace public.

34. Notamment s'agissant du droit de l'acquisition de la nationalité française (CE, 27 juin 2008, n² 286798, Mabchour, AJDA 2008, p. 2013, note Chrestia P.), de la fermeture des mosquées (CE, ord., 6 déc. 2016, n 405476, Assoc. islamique Malik Ibn Anas; CE, 20 janv. 2017, $\mathrm{n}^{\circ}$ 406618, Assoc. Centre culturel franco-égyptien - l'association Maison d'Égypte), et d'assignations à résidence (CE, ord,. 25 avril 2017, n 409725, M. D.). 
contre la radicalisation fait actuellement l'objet d'une politique publique, mais principalement à travers le prisme de la lutte contre le terrorisme ${ }^{35}$; or les individus retenant une lecture intégriste de leur religion ne sont évidemment pas tous dangereux.

La seconde question consiste à se demander dans quelle mesure les normes juridiques peuvent intégrer des normes de comportement. Le critère qui semble décisif est celui du bon fonctionnement du service public, voire celui de l'ordre public. Si on peut trouver acceptable le fait de ne pas recruter des personnes qui vont entraver le bon fonctionnement du service public en s'isolant et en refusant toute interaction sociale avec les individus de sexe opposé, on constate que les notions de bon fonctionnement du service public et d'ordre public sont mouvantes et font l'objet d'interprétation extensive. En la matière, l'objectivité ne semble pas de mise.

La troisième interrogation consiste à se demander si les membres d'un jury peuvent se fonder sur les intentions d'un candidat pour rejeter sa candidature. Le jury doit-il se cantonner à l'évaluation des compétences des candidats ou élargir ses attributions à la prévention des risques de troubles au bon fonctionnement du service public ? Peut-il légalement écarter une candidature en se fondant sur un comportement qui n'a pas encore eu lieu ? On pourrait en effet concevoir que le jury se borne à l'évaluation des compétences des candidats et que les agents publics ne soient sanctionnés qu'à la suite d'un comportement inadéquat.

Par ailleurs, il ne faudrait pas que la vigilance à l'égard de la lecture intégriste de l'islam d'un nombre restreint de musulmans ait des répercussions défavorables sur l'ensemble des personnes de confession musulmane. On constate pourtant que l'obligation de neutralité religieuse, telle qu'elle est interprétée depuis une quinzaine d'années, s'avère davantage préjudiciable aux musulmans, y compris aux non-intégristes.

35. V. la loi n $2014-1353$ du 13 novembre 2014 renforçant les dispositions relatives à la lutte contre le terrorisme et la circulaire NOR-INTK1405276C du 29 avril 2014 du ministre de l'Intérieur relative à la prévention de la radicalisation. 


\section{UNE INTERPRÉTATION EXTENSIVE DE L'OBLIGATION DE NEUTRALITÉ RELIGIEUSE DES AGENTS PUBLICS DAVANTAGE PRÉJUDICIABLE AUX MUSULMANS}

On se propose ici de présenter l'interprétation jurisprudentielle extensive de l'obligation de neutralité religieuse des agents publics (2.1), puis de s'interroger sur les justifications de la différence de traitement engendrée par le devoir de neutralité religieuse (2.2).

\subsection{FONDEMENTS ET MISE EN CEUVRE DE L'OBLIGATION DE NEUTRALITÉ RELIGIEUSE DES AGENTS PUBLICS}

L'obligation de neutralité religieuse est évidemment liée au principe de laïcité qui, rappelons-le, a valeur constitutionnelle. Celle-ci fait obstacle à ce que les agents publics disposent, dans le cadre de leurs fonctions, du droit de manifester leurs croyances religieuses. Le premier arrêt du Conseil d'État à se référer explicitement à ce devoir est l'arrêt Delle Jamet du 3 mai $1950^{36}$ : il y est affirmé que, dans l'accomplissement de leurs fonctions, les agents publics doivent respecter le « devoir de stricte neutralité qui s'impose à tout agent collaborant à un service public ».

Jusqu'en 2000, les litiges concernaient essentiellement des enseignants ou des fonctionnaires en contact avec les usagers. Or, dans l'avis du 3 mai 2000 Mlle Marteaux ${ }^{37}$, le Conseil d'État précise que l'obligation de neutralité s'impose à tous les agents, quels que soient leur statut, le service où ils sont affectés ou la fonction exercée et qu'ils soient ou non en contact avec le public : il indique, d'une part, que « le fait pour un agent $[\ldots]$ de manifester dans l'exercice de ses fonctions ses croyances religieuses, notamment en portant un signe destiné à marquer son appartenance à une religion, constitue un manquement à ses obligations » mais affirme, d'autre part, que « les suites à donner à ce manquement, notamment sur le plan disciplinaire, doivent être appréciées par l'administration sous le contrôle du juge, compte tenu de la nature et du degré de caractère ostentatoire de ce signe, comme des autres circonstances dans lesquelles le manquement est constaté ».

36. CE, 3 mai 1950, n 28238, Delle Jamet, Rec. CE, p. 247, S. 1951, 3, 73.

37. CE, avis, 3 mai 2000, n 217077, Mlle Marteaux, AJDA 2000, p. 673, chron. GuYOMAR M. et Colin P. 
La neutralité religieuse interdit donc aux agents publics de porter une tenue ou un signe destiné à marquer leur appartenance à une religion. À cet égard, de nombreux arrêts confirment les sanctions prises à l'encontre de femmes portant le foulard islamique ${ }^{38}$, ou même un simple bandana ${ }^{39}$, dont le « caractère ostentatoire » n'est pourtant pas évident. Dans l'arrêt du 26 novembre 2015 Ebrahimian c/ France ${ }^{40}$, la Cour européenne des droits de l'homme a, quant à elle, estimé que l'interdiction du port du voile par les agents publics ne constituait pas une violation de l'article 9 de la Convention européenne des droits de l'homme.

L'obligation de neutralité religieuse interdit également le prosélytisme, c'est-à-dire le fait pour une personne de chercher à rallier d'autres individus à sa religion. S'agissant d'un agent qui remettait des imprimés à caractère religieux aux usagers, le Conseil d'État a jugé que ces actes de prosélytisme constituaient une faute susceptible de perturber la bonne marche du service et contraire à l'honneur professionnel ${ }^{41}$.

Par ailleurs, dans l'arrêt Odent de $2003^{42}$, le Conseil d'État a considéré que constituait un manquement à l'obligation de neutralité le fait pour un agent public d'utiliser les moyens de son service pour communiquer avec une secte et d'apparaître sur le site public de cette secte en qualité de membre, en mentionnant son adresse électronique professionnelle. En revanche, le juge administratif estime qu'en l'absence de tout comportement incompatible avec l'exercice des fonctions, l'appartenance d'un agent public à un groupe sectaire ne constitue pas une faute ${ }^{43}$.

Il est permis de se demander s'il est pertinent de sanctionner de la même façon les actes de prosélytisme et le port d'un signe ou vêtement destiné

38. CAA Lyon, 27 nov. 2003, n 03LY01392, Ben Abdallah, RFDA 2004, p. 588, concl. Kolbert E., s'agissant d'une contrôleuse du travail ; CAA Versailles, 23 févr. 2006, n 04VE03227, Mme A., AJDA 2006, p. 1237, s'agissant d'une assistante maternelle licenciée alors qu'elle était enceinte; TA Toulouse, ord., 17 avril 2009, S. Trojet, s'agissant d'une doctorante allocataire de recherche ; CAA Versailles, 21 mars 2013, n 11VE00853, Mme B., s'agissant d'une agente administrative des services fiscaux.

39. CAA Versailles, 6 oct. 2011, n 09VE02048, Mme Meryem A., s'agissant d'une assistante maternelle.

40. CEDH, 26 nov. 2015, n 64846/11, Ebrahimian c/ France, AJFP 2016, p. 32, note ZARCA A.

41. CE, 19 févr. 2009, n 311633, M. B., AJDA 2009, p. 340, note De Montecler M.-C.; AJFP 2009, p. 253, concl. Bourgeois-MAChureau B. - V. également CAA Versailles, 30 juin 2016, $\mathrm{n}^{\circ}$ 15VE00140, M. C.

42. CE, 15 oct. 2003, n 244428, Odent, Rec. CE, p. 402, AJFP 2004, p. 31, note Guillaumont O.

43. V. par ex. TA Melun, 15 févr. 2005, n 01-3630-5, Melle L., AJFP 2005, p. 317, concl. DALVI ou CAA Lyon, 4 oct. 2005, n 99LY00612, Ministre de l'Éducation nationale c/ M. T. 
à exprimer une conviction religieuse. Cela ne contribue-t-il pas à amalgamer la manifestation d'une appartenance religieuse et la volonté d'imposer ses croyances à autrui ? Au vu de la jurisprudence concernant des agents portant un tel signe, le devoir de neutralité a des conséquences négatives essentiellement sur les agents publics musulmans. La plupart des arrêts concernent ainsi des agentes voilées et jugent légales les décisions de licenciement les concernant, le port d'un voile ou d'un bandana étant considéré comme une "faute grave », au même titre que les actes de prosélytisme ${ }^{44}$. On ne peut en revanche prouver la discrimination directe ${ }^{45}$ à l'égard des musulmans dans la mesure où, à notre connaissance, aucun arrêt comparable ne concerne des agents portant un autre signe religieux. Il est pourtant permis de se questionner sur la proportionnalité de la sanction et de se demander si un agent portant une croix serait sanctionné de la même façon. L'absence de jurisprudence en la matière ne signifie pas en effet que de telles situations n'existent pas; elles sont peut-être mieux acceptées par les collègues et les supérieurs hiérarchiques et ne génèrent pas de contentieux. Surtout, le fait que l'avis Mlle Marteaux ait fait du " degré de caractère ostentatoire » un élément déterminant pour apprécier la sanction a, selon nous, introduit un désavantage au détriment des personnes de confession musulmane, une croix étant considérée comme un signe présentant un degré d'ostentation inférieur à un foulard. Le fait même d'avoir introduit cet élément semble participer d'une volonté de sanctionner plus fortement le port du voile.

On aurait pu penser que les nouveaux textes renforçant le devoir de neutralité religieuse en uniformisent l'application, en ne se référant plus à cette « circonstance $^{46} »$. En effet, si on peut concevoir que la neutralité politique soit appliquée plus ou moins strictement selon les fonctions occupées par les agents publics, rien ne justifie que le port d'un signe religieux visible soit sanctionné plus ou moins sévèrement. Or, la circulaire du 15 mars 2017 indique qu'«En cas de poursuite disciplinaire, la sanction prononcée doit,

44. Le rapport annuel 2015-2016 de l'Observatoire de la laïcité affirme également que « la manifestation d'une opinion religieuse (prosélytisme et expression des convictions religieuses) sur le lieu de travail ou dans l'exercice des fonctions n'est pas autorisée (sanction disciplinaire) », op. cit., p. 110. Les agents publics informent pourtant indirectement leur chef de service de leur confession lorsqu'ils font la demande d'une autorisation d'absence pour une fête religieuse non intégrée dans le calendrier.

45. Définie par la loi du 27 mai 2008 comme « la situation dans laquelle, sur le fondement [d'un critère prohibé], une personne est traitée de manière moins favorable qu'une autre ne l'est, ne l'a été ou ne l'aura été dans une situation comparable».

46. Le rapport du Conseil d'État Un siècle de laïcité évoque un «tempérament apporté à cette règle stricte », Rapport public 2004, Paris, La Documentation française, 2004, p. 275. 
sous le contrôle normal du juge, être proportionnée à la faute et tenir compte des circonstances de l'espèce telles que la nature des fonctions exercées, la réitération du manquement malgré des rappels à la règle, le degré d'ostentation du signe d'appartenance religieuse, le port de ce signe au contact ou non du public, la vulnérabilité du public, etc. (V. par ex., CAA Versailles, 23 février 2006, n 04VE03227) ${ }^{47}$ ». Entérine-t-elle dès lors une obligation de neutralité religieuse à géométrie variable? Questionner cette différence de traitement est, pour nous, l'occasion de revenir sur les fondements du devoir de neutralité religieuse.

\subsection{COMMENT JUSTIFIER LA DIFFÉRENCE DE TRAITEMENT DES AGENTS PUBLICS FONDÉE SUR L'OBLIGATION DE NEUTRALITÉ RELIGIEUSE ?}

L'obligation de neutralité religieuse serait fondée sur la laiicité, plus particulièrement sur la séparation des Églises et de l'État énoncée par la loi du 9 décembre $1905^{48}$. En vertu de ce texte, l'État se doit d'être neutre vis-à-vis des religions, il ne doit pas intervenir dans ce domaine qui doit relever de la sphère privée de chacun; parallèlement, les religions ne s'immiscent pas dans le fonctionnement des pouvoirs publics. C'est donc en tant que représentants de l'État ou d'une autre collectivité publique que les agents publics doivent apparaître neutres.

Si le devoir de neutralité religieuse des agents publics découle directement de la séparation des Églises et de l'État, on a également coutume de le relier au principe d'égalité. Plus précisément, il est communément admis que la neutralité du service public constitue un corollaire du principe d'égalité ${ }^{49}$. On considère en effet que les agents publics ne doivent privilégier ni discriminer aucun usager et que le port d'un signe religieux serait source d'une potentielle différence de traitement. À notre connaissance, peu de juristes ont analysé cette affirmation : il est pourtant permis de s'interroger sur le point de savoir si les agents publics d'une certaine religion vont favoriser

47. Souligné par nous. Se référer à l'arrêt concernant le licenciement, pour port du voile, d'une femme enceinte n'est peut-être pas le meilleur exemple en termes de proportionnalité. Le fait de distinguer si le signe est porté en contact ou non du public s'éloigne par ailleurs de l'avis Mlle Marteaux.

48. Loi du 9 décembre 1905 concernant la séparation des Églises et de l'État.

49. Cons. const., déc. 18 sept. 1986, n 86-217 DC, cons. 15 relatif au pluralisme dans les services de communication. Il n'est toutefois pas question de neutralité des agents publics. En outre, contrairement à ce qu'on peut lire, la décision n 96-380 DC du 23 juillet 1996 n'affirme pas expressément que la neutralité fait partie des principes constitutionnels régissant le service public, V. par ex. Conseil d'État, Rapport public 2004, op. cit., p. 275. 
les usagers pratiquant la même religion et discriminer les autres. Même en admettant ce postulat discutable, on peut se demander en quoi un fonctionnaire portant un signe religieux favoriserait ou discriminerait davantage les usagers en fonction de leur religion qu'un fonctionnaire n'en portant pas. En effet, si on suppose qu'il peut y avoir une connivence entre agents publics et usagers de même religion, en quoi le fait de porter un signe religieux y contribuerait et en quoi le fait de ne pas en porter permettrait de l'éviter ?

Aussi, selon nous, le devoir de neutralité des agents publics se fonde uniquement sur la séparation des Églises et de l'État : c'est parce qu'ils incarnent une institution publique que les agents publics ne peuvent pas porter de signes religieux. Autrement dit, dans la mesure où les personnes publiques sont soumises à un devoir de neutralité, les usagers du service public ne doivent pas savoir de quelle religion relèvent les agents publics. On peut alors se questionner sur ce qui justifie qu'un agent public qui n'est pas en contact avec le public soit licencié s'il arbore un signe religieux. La question de la modulation de l'obligation de neutralité selon le type de fonctions exercées par les agents publics mériterait, à ce titre, d'être posée car elle serait susceptible de concilier davantage le devoir de neutralité confessionnelle et le principe de non-discrimination à raison de la religion ${ }^{50}$. On rétorquera alors qu'il ne s'agit pas d'une simple «image de marque » de l'administration et qu'il est préférable que l'ensemble des agents publics respectent tous ce devoir, d'une part car ils relèvent d'une personne publique, elle-même soumise à l'obligation de neutralité et, d'autre part, car la distinction des devoirs en fonction des postes est susceptible d'engendrer un certain nombre d'effets pervers. Un tel raisonnement ne se justifie cependant que si le devoir de neutralité religieuse s'applique strictement de la même manière à tous les agents publics.

S'il est difficile de prouver la différence de traitement entre agents de confession musulmane et agents d'autres confessions, il est permis de douter que le juge administratif appliquerait aujourd'hui à un imam la solution que le Conseil d'État avait admise dans un avis de $1972^{51}$ s'agissant d'un ecclésiastique : s'éloignant de la jurisprudence Abbé Bouteyre, il avait affirmé que «si les dispositions constitutionnelles qui ont établi la laïcité de l'État et celle de l'enseignement imposent la neutralité de l'ensemble des services publics et en particulier la neutralité du service de l'enseignement à l'égard

50. Tel est le raisonnement de la CJUE dans l'affaire C-157/15 du 14 mars 2017, Samira Achbita c/ G4S Secure Solutions NV.

51. CE, avis, 21 sept. 1972, n 309-354, Laïcité du corps enseignant, GACE 2002, nº 6. 
de toutes les religions, elles ne mettent pas obstacle par elles-mêmes à ce que des fonctions de ces services soient confiées à des membres du clergé ». L'état ecclésiastique ne serait donc pas, par lui-même, incompatible avec la qualité d'enseignant, mais le fait qu'une doctorante allocataire de recherche porte un voile serait constitutif d'une faute grave...

On constate d'ailleurs que les administrations et le juge administratif sont plus exigeants à l'égard des femmes que des hommes. En effet, alors que les arrêts rejetant les recours à l'encontre de décisions de licenciement en raison de port du voile sont fréquents, ceux concernant un licenciement pour cause de barbe fournie sont rares ${ }^{52}$. Sur ce point, il ne nous semble pas évident que le port du foulard islamique soit, par nature, ostentatoire. C'est ainsi que, dans une décision du 27 janvier $2015^{53}$, la première chambre de la Cour constitutionnelle fédérale allemande a considéré qu'une interdiction de principe du port du foulard par les enseignants dans les écoles publiques ouvertes au plan confessionnel était contraire à la Loi fondamentale. Il est en outre permis de se demander en quoi la révocation des femmes portant un simple foulard contribue au "vivre ensemble ». N'est-il pas, au contraire, susceptible d'engendrer un repli communautaire?

En France, la tendance actuelle semble pourtant pencher en faveur d'un alignement du droit du travail sur le droit de la fonction publique, et donc d'une généralisation du devoir de neutralité religieuse, alors même que la séparation des Églises et de l'État ne peut justifier, en droit du travail, une telle obligation ${ }^{54}$. La rédaction de l'article L. 1321-2-1 du Code du travail telle qu'elle résulte de la loi Travail du 8 août $2016^{55}$ illustre bien ce mouvement. Celui-ci dispose en effet que « Le règlement intérieur peut contenir des dispositions inscrivant le principe de neutralité et restreignant la manifestation des convictions des salariés si ces restrictions sont justifiées par l'exercice d'autres libertés et droits fondamentaux ou par les nécessités du bon fonctionnement de l'entreprise et si elles sont proportionnées au

52. V. néanmoins CAA Versailles, 19 févr. 2008, n 06VE02005, Commune de Villemomble; BUI-XUAN O., "Regard genré sur les dispositions juridiques relatives à la neutralité religieuse ", in Hennette Vauchez S., Roman D. et Möschel M. (dir.), Ce que le genre fait au droit, Paris, Dalloz, 2013, p. 25.

53. 1 BvR 471/10 ; V. Rabault H., "Le droit des enseignantes à arborer le foulard », RFD const. 2015, p. 256.

54. Selon Yannick Pagnerre, en ce qui concerne la neutralité dans l'entreprise, « la laïcité apparaît comme un faux fondement », PAgnerRe Y., " Principe de neutralité dans l'entreprise », Dr. soc. 2016, p. 881.

55. Loi $\mathrm{n}^{\circ}$ 2016-1088 du 8 août 2016 relative au travail, à la modernisation du dialogue social et à la sécurisation des parcours professionnels. 
but recherché ». L'obligation de neutralité religieuse, qui était auparavant l'exception dans le secteur privé, est donc susceptible d'être généralisée aux entreprises. S'inscrivant dans le contexte post-attentats, les débats parlementaires relatifs à cette disposition ont également évoqué la lutte contre le communautarisme ${ }^{1}$. Aussi, tout comme l'intégration de l'obligation de neutralité dans le statut général des fonctionnaires, la rédaction de cet article relève d'une volonté politique de prévenir (et de lutter contre) la radicalisation islamiste. Mais il n'a pas été question d'opérer de distinctions entre radicalisation islamiste, intégrisme musulman, communautarisme et port du voile. Tout au contraire, le trouble introduit par la loi du 11 octobre 2010 interdisant la dissimulation du visage dans l'espace public semble conforté. Le fait que la loi «anti-burqa » ait, s'agissant d'une pratique extrémiste, substitué « l'espace public ${ }^{2}$ », à la "sphère publique » - périmètre traditionnel de la laïcité - a, selon nous, donné lieu à une dilatation progressive du champ d'application du devoir de neutralité religieuse des individus ${ }^{3}$ : celui-ci a d'abord été étendu aux salariés travaillant dans des structures de droit privé accomplissant une mission de service public ${ }^{4}$ et, aujourd'hui, aux salariés de droit privé travaillant dans les entreprises. Dans un arrêt du 14 mars 2017, la Cour de justice de l'Union européenne a également jugé que le règlement intérieur d'une entreprise interdisant le port visible de tout signe religieux ne constituait pas une discrimination directe ${ }^{5}$. Toutefois, une

1. Lemoyne J.-B., Gabouty J.-M. et Forissier M., Rapport sur le projet de loi visant à instituer de nouvelles libertés et de nouvelles protections pour les entreprises et les actif-ve-s, Paris, Sénat,

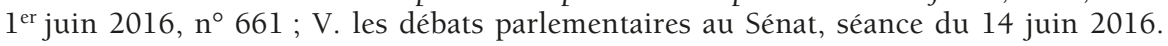

2. V. Bui-Xuan O., «L'espace public. L'émergence d'une nouvelle catégorie juridique? Réflexions sur la loi interdisant la dissimulation du visage dans l'espace public», RFDA 2011, p. 551 ; DieU F., « Laïcité et espace public », RDP 2013, p. 566.

3. Bui-Xuan O., "Espace public et libertés religieuses », in Bui-Xuan O. (dir.), Droit et espace(s) public(s), Clermont-Ferrand, Fondation Varenne, 2012, p. 123.

4. Cass. soc., 19 mars 2013, $\mathrm{n}^{\circ}$ 11-28.845, Mme X. c/ Association Baby-Loup, et Cass. soc., 19 mars 2013, n 12-11.690, Mme X. c/ Caisse primaire d'assurance maladie de Seine-SaintDenis, AJDA 2013, p. 1069, note DreYFus J.-D.

5. CJUE, Gde ch., 14 mars 2017, aff. C-157/15, Samira Achbita c/ G4S Secure Solutions NV, s'agissant d'une entreprise belge qui avait modifié son règlement intérieur afin d'interdire aux travailleurs de porter sur le lieu de travail des signes visibles de leurs convictions ou d'accomplir tout rite en découlant, après qu'une salariée ait fait savoir qu'elle souhaitait porter le foulard islamique. Dans la seconde affaire du même jour (CJUE, Gde ch., 14 mars 2017, aff. C-188/15, Asma Bougnaoui c/ Micropole SA,) qui concernait une salariée française licenciée après avoir refusé d'ôter son voile à la suite de la plainte d'un client, la Cour a affirmé que « la volonté d'un employeur de tenir compte des souhaits d'un client de ne plus voir les services dudit employeur assurés par une travailleuse portant un foulard islamique ne saurait être considérée comme une exigence professionnelle essentielle et déterminante au sens de la directive». 
telle règle interne est susceptible de constituer une discrimination indirecte, à moins qu'elle ne soit « objectivement justifiée par un objectif légitime, tel que la poursuite par l'employeur, dans ses relations avec les clients, d'une politique de neutralité politique, philosophique ainsi que religieuse, et que les moyens de réaliser cet objectif ne soient appropriés et nécessaires ${ }^{6}{ }$.

Alors que le Conseil d'État a récemment affirmé que les crèches de Noël pouvaient ne pas avoir de "signification religieuse particulière » et, dans certaines circonstances, être installées par une personne publique dans un emplacement public ${ }^{7}$, on constate que l'obligation de neutralité religieuse à laquelle n'étaient initialement soumis que les agents publics tend à s'appliquer à un nombre croissant de personnes, lesquelles voient mécaniquement leur liberté d'expression religieuse se restreindre. La neutralité religieuse constituerait-elle dès lors une obligation à plusieurs vitesses?

En apparence universaliste et neutre, le devoir de neutralité confessionnel ne génère-t-il pas, en fait, un désavantage particulier pour les personnes de confession musulmane? La plupart des affaires évoquées nous conduisent à le penser mais, comme l'indique Alexis Zarca, les agents publics « requérants n'ont que très rarement invoqué le moyen tiré de la discrimination indirecte dans l'exercice de la religion, au titre de ce que l'interdiction aurait vocation à frapper davantage, de fait, les agents de certaines confessions ${ }^{8} »$. Reste à savoir si les différences de traitement sont objectivement justifiées par un motif légitime et si les moyens de réaliser cet objectif sont proportionnés. La marge d'interprétation semble ici importante, le contrôle de proportionnalité du principe de neutralité religieuse pouvant «aboutir à des solutions diamétralement différentes ${ }^{9} »$.

6. CJUE, Gde ch., 14 mars 2017, aff. C-157/15.

7. CE, ass., 9 nov. 2016, n 395122, Fédération départementale des libres penseurs de Seineet-Marne; $\mathrm{n}^{\circ}$ 395223, Fédération de la libre pensée de Vendée. Pour une lecture critique, V. Slama S., "Jésus revient au Palais Royal ou quand le Conseil d'État fait obstacle à la séparation de l'État et de l'étable », La Revue des droits de l'homme, nº 11, 2017.

8. ZARCA A., op. cit., p. 720.

9. Pagnerre Y., op. cit., p. 885. 